\title{
A Clinical Case of Scrub Typhus in the United States Forces Korea Patient with Eschar and Genetic Identification of Orientia tsutsugamushi Using Multiplex PCR-Based Next-Generation Sequencing
}

\author{
Seungchan Cho ${ }^{1,+}{ }^{\text {, Jon C. Allison }}{ }^{2,+}, \ddagger$, Kkothanahreum Park ${ }^{1}$, Jin Sun No ${ }^{1}$, Seung-Ho Lee ${ }^{1}$, Kyungmin Park ${ }^{1} \mathbb{D}$, \\ Jongwoo Kim ${ }^{1}$, Terry A. Klein ${ }^{2}$, Heung-Chul Kim ${ }^{2}$ (D, Won-Keun Kim ${ }^{3,4}$ (D) and Jin-Won Song ${ }^{1, *}$ \\ check for \\ updates \\ Citation: Cho, S.; Allison, J.C.; Park, \\ K.; No, J.S.; Lee, S.-H.; Park, K.; Kim, \\ J.; Klein, T.A.; Kim, H.-C.; Kim, W.-K.; \\ 1 Department of Microbiology, College of Medicine, Korea University, Seoul 02841, Korea; \\ schanchan@korea.ac.kr (S.C.); pkhar@korea.ac.kr (K.P.); njs2564@gmail.com (J.S.N.); \\ leeds1104@korea.ac.kr (S.-H.L.); kmpark0131@korea.ac.kr (K.P.); hotdog442@korea.ac.kr (J.K.) \\ 2 Force Health Protection and Preventive Medicine, MEDDAC-Korea, 65th Medical Brigade, Unit 15281, \\ APO AP 96251-5281, USA; jon.c.allison.mil@mail.mil (J.C.A.); terry.a.klein2.civ@mail.mil (T.A.K.); \\ hungchol.kim2.ln@mail.mil (H.-C.K.) \\ 3 Department of Microbiology, College of Medicine, Hallym University, Chuncheon 24252, Korea; \\ wkkim1061@hallym.ac.kr \\ 4 Institute of Medical Science, College of Medicine, Hallym University, Chuncheon 24252, Korea \\ * Correspondence: jwsong@korea.ac.kr; Tel.: +82-2-920-6353; Fax: +82-2-923-3645 \\ + The authors have equally contributed. \\ $\ddagger$ Current address: Department of Preventive Medicine, MEDDAC-Bavaria, Unit 28037, \\ APO AE 09112-8037, USA.
} et al. A Clinical Case of Scrub Typhus in the United States Forces Korea Patient with Eschar and Genetic Identification of Orientia tsutsugamushi Using Multiplex PCR-Based Next-Generation Sequencing. Pathogens 2021, 10, 424. https://doi.org/10.3390/ pathogens 10040424

Academic Editors: Jose Muñoz Gutiérrez and Lawrence S. Young

Received: 21 January 2021

Accepted: 26 March 2021

Published: 2 April 2021

Publisher's Note: MDPI stays neutral with regard to jurisdictional claims in published maps and institutional affiliations.

Copyright: (c) 2021 by the authors. Licensee MDPI, Basel, Switzerland. This article is an open access article distributed under the terms and conditions of the Creative Commons Attribution (CC BY) license (https:// creativecommons.org/licenses/by/ $4.0 /)$.
Abstract: An epidemiological investigation was conducted for a scrub typhus case reported in a U.S. Forces Korea military patient in the Republic of Korea in November 2018. The patient had a fever, maculopapular rash, and an eschar. The full-length sequence of Orientia tsutsugamushi 56-kDa type-specific antigen (TSA) gene was obtained from eschar tissue by multiplex PCR-based Next Generation Sequencing for genetic identification. Based on the 56-kDa TSA gene, the O. tsutsugamushi aligned most closely with the Boryong strain.

Keywords: Orientia tsutsugamushi; scrub typhus; genetic identification; target enrichment nextgeneration sequencing

\section{Introduction}

Scrub typhus is an acute febrile bacterial disease caused by the arthropod-borne gramnegative bacillus, Orientia tsutsugamushi, commonly identified in rural areas. Scrub typhus is endemic to the Asia-Pacific region, extending from Afghanistan to Korea, China, the islands of the Southwestern Pacific, and Northern Australia, and might also be present in Africa and South America [1-3]. In recent years, there have been approximately 8000 clinical cases of scrub typhus annually in Korea, with a fatality rate of $1.32 \%$ with good medical care [4]. Typically, clinical manifestation and signs develop within one to two weeks after infection and are characterized by fever, headache, muscle pain, cough, gastrointestinal upset, maculopapular rash, and eschar. Recently, the genetic analysis of O. tsutsugamushi has been used to establish and differentiate genotypes [5]. More than 20 antigenic variants of $O$. tsutsugamushi have been reported worldwide, including Boryong, Kuroki, Gilliam, Kato, Karp, and Kawasaki strains.

The immune-dominant $56-\mathrm{kDa}$ type-specific antigen (TSA) is located on the surface of the bacteria membrane [6]. The 56-kDa TSA is not expressed in other bacteria, including other members of the Rickettsiaceae. Other genes may be useful for studies of differentiation within Orientia strains, but to date, no other locus seems to have the same potential of 
usefulness for analysis of strain variation [5]. Compared to the genes for 16S rRNA or groEL, the high level of variability of 56-kDa TSA appears to be a representative marker gene for differentiating O. tsutsugamushi strains [7]. Target-enrichment nextgeneration sequencing (NGS) methods exhibited the effective recovery of viral genome sequences from rodent tissue containing low copies of viral RNA [8,9]. To investigate an extremely low amount of genome sequences of $O$. tsutsugamushi from the patient's tissue (eschar) with scrub typhus, we performed multiplex PCR-based NGS for the enrichment of the 56-kDa TSA gene. The obtained full-length sequences allowed the genetic identification of O. tsutsugamushi genotypes. Phylogenetic analysis of the full-length 56-kDa gene demonstrated that the United States Forces Korea (USFK) patient was infected with O. tsutsugamushi that aligned with the Boryong strain. The clinical aspect of the scrub typhus USFK patient and genetic characterization of $O$. tsutsugamushi raises awareness for the surveillance of an endemic zoonotic disease, scrub typhus, in the ROK.

\section{Results}

From 3-4 November 2018, a 46-year-old male and his family visited Jirisan National Park located at the southern tip of the Korean Peninsula. He visited a gorge, temple site, and garden area where he walked along trails in a forested area with cut/uncut grasses/herbaceous vegetation. On 12 November 2018, the patient noted a nickel-sized black scab on his lower left calf of his leg without other symptoms. On 14 November, the patient felt very tired after running and a day later experienced generalized myalgia with mild intermittent headaches and sweats (Table 1). He took Ibuprofen for pain relief (PO 200-400 mg PRN).

On 16 November 2018, the patient reported to the Jenkins Army Health Clinic, Camp Humphreys, with a chief complaint of a "bug bite" (eschar) about the size of a nickel with a localized inflammatory reaction on his lower left calf (Figure 1A). He reported myalgia with mild intermittent headaches, chills, and sweats. He was prescribed acetaminophen, PO $325 \mathrm{mg}$, 1 to 2 tablets every $4 \mathrm{~h}$ as needed for pain or fever. On 17-18 November, he continued to have intermittent headaches, chills, and night sweats.

On 19 November 2018, the patient noted a body rash and reported to the Jenkins Army Health Clinic with worsening symptoms of intermittent dizziness, fever, chills, night sweats, fatigue, and loss of appetite. He noted a "non-itchy" maculopapular rash that developed over the entire body $24 \mathrm{~h}$ prior to the visit (Figure 1B). He was diagnosed with an insect bite (nonvenomous) that was red and raised with a dark center on his left lower leg and pruritus and a maculopapular rash of unclear etiology. The patient was prescribed sulfamethoxazole/trimethoprim $(800 \mathrm{mg} / 160 \mathrm{mg}), 1$ tablet every $12 \mathrm{~h}$, to treat a presumptive bacterial infection. On the following day (20 November), he noted that the rash had spread from the body to the legs. On 21 November, the patient indicated that he felt better, but the body rash increased.

On 21 November, the patient was referred to the Saint Mary's Hospital, Pyeongtaek where he was admitted with fever, chills, myalgia, maculopapular rash (head, trunk), sweats, and eschar, that had been presumptively diagnosed as an insect/spider bite. Based on the symptoms, the patient was presumptively diagnosed with scrub typhus and prescribed doxycycline, $100 \mathrm{mg} 3$ times daily after a meal for treatment of O. tsutsugamushi, acetaminophen $650 \mathrm{mg}$ for pain, acetylcysteine $200 \mathrm{mg}$ to reduce liver damage resulting from extensive use of acetaminophen, ursodeoxycholic acid $250 \mathrm{mg}$ to reduce liver damage, Godex $150 \mathrm{mg}$ capsule (carnitine orotate $150 \mathrm{mg}$, 17 amino acids, $12.5 \mathrm{mg}$; pyridoxine $\mathrm{HCl}$, $25 \mathrm{mg}$; adenine $\mathrm{HCl}, 2.5 \mathrm{mg}$, cyanocobalamin $125 \mu \mathrm{g}$, and riboflavin, $500 \mu \mathrm{g}$ ) to reduce liver enzyme level, and rebamipide $100 \mathrm{mg}$ to provide gastrointestinal protection. 
Table 1. Clinical signs and symptoms of the United States Forces Korea (USFK) patient infected by O. tsutsugamushi.

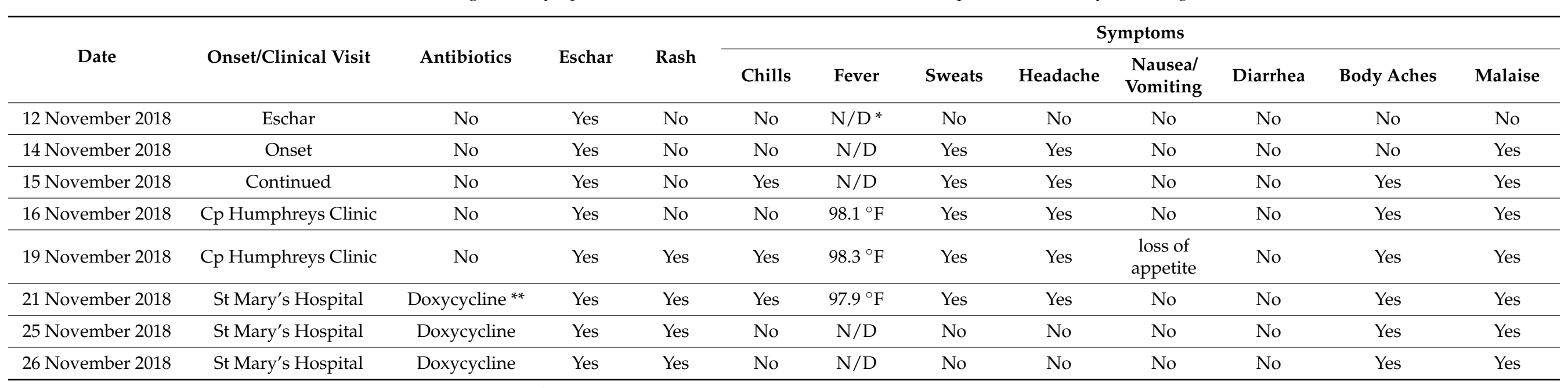

${ }^{*} \mathrm{~N} / \mathrm{D}$, not determined. ${ }^{* *}$ Doxycycline $(100 \mathrm{mg})$ was prescribed twice daily. 

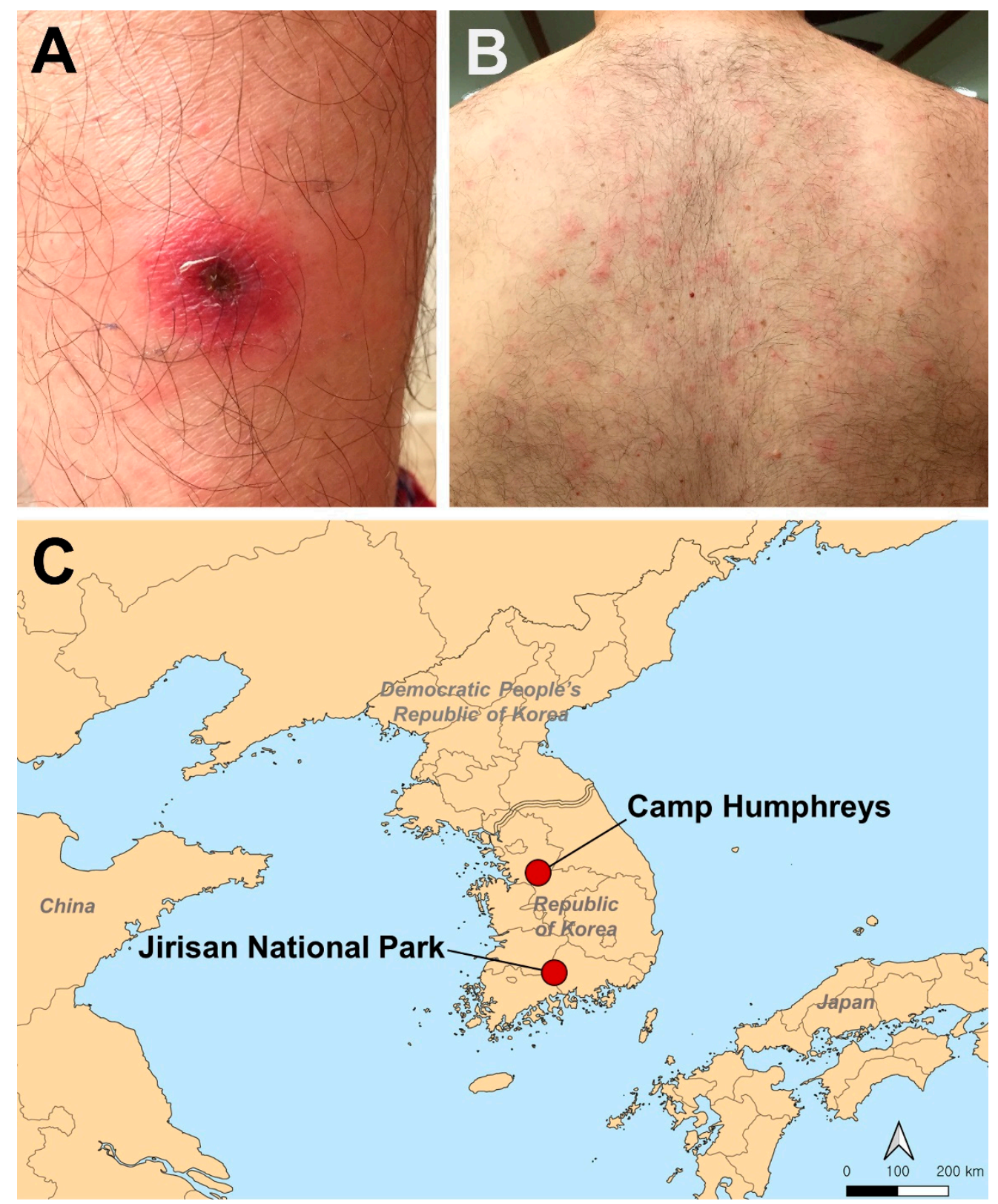

Figure 1. An eschar on the lower left lower calf and maculopapular rash on the back of the USFK patient with Orientia tsutsugamushi infection. (A) Eschar location; (B) Back with maculopapular rash; (C) The location of Camp Humphreys and Jirisan National Park, Republic of Korea.

On 26 November, the patient was interviewed by Force Health Protection and Preventive Medicine, 65th Medical Brigade, and a biopsy of the eschar was obtained with the patient's verbal approval. The eschar tissue was placed in a $-80^{\circ} \mathrm{C}$ freezer and transported to Korea University the following day. The patient provided photos of the rash and eschar, as well as area information of the military base where he worked and Jirisan National Park that he visited (Figure 1C). On 28 November, the fever had subsided, and the patient was discharged from the hospital.

We extracted DNA from the eschar and performed nested PCR (the expected size of an amplicon, $632 \mathrm{bps}$ ) using specific primers for the 56-kDa TSA (1599 bps) of O. tsutsugamushi. The phylogeny of the 56-kDa TSA nucleotide sequence formed a close genetic lineage with the Boryong genotype, showing $99.8 \%(631 / 632)$. The amino acid sequence of 56-kDa TSA also showed a high homology of $99.5 \%$ with the Boryong genotype. 
To obtain the full-sequence of 56-kDa TSA gene from the patient's eschar tissue containing an extremely low amount of the $O$. tsutsugamushi genome, we designed multiplex PCR primers with 150 or 300 bps interval and amplified the targeted gene as shown (Table 2) and performed the NGS using MiSeq Benchtop. The complete genomic sequence of 56-kDa TSA from the patient was inferred to a phylogenetic group belonging to the Boryong strain (Figure 2). The genomic sequence was deposited in the GenBank (accession number: MT989475).

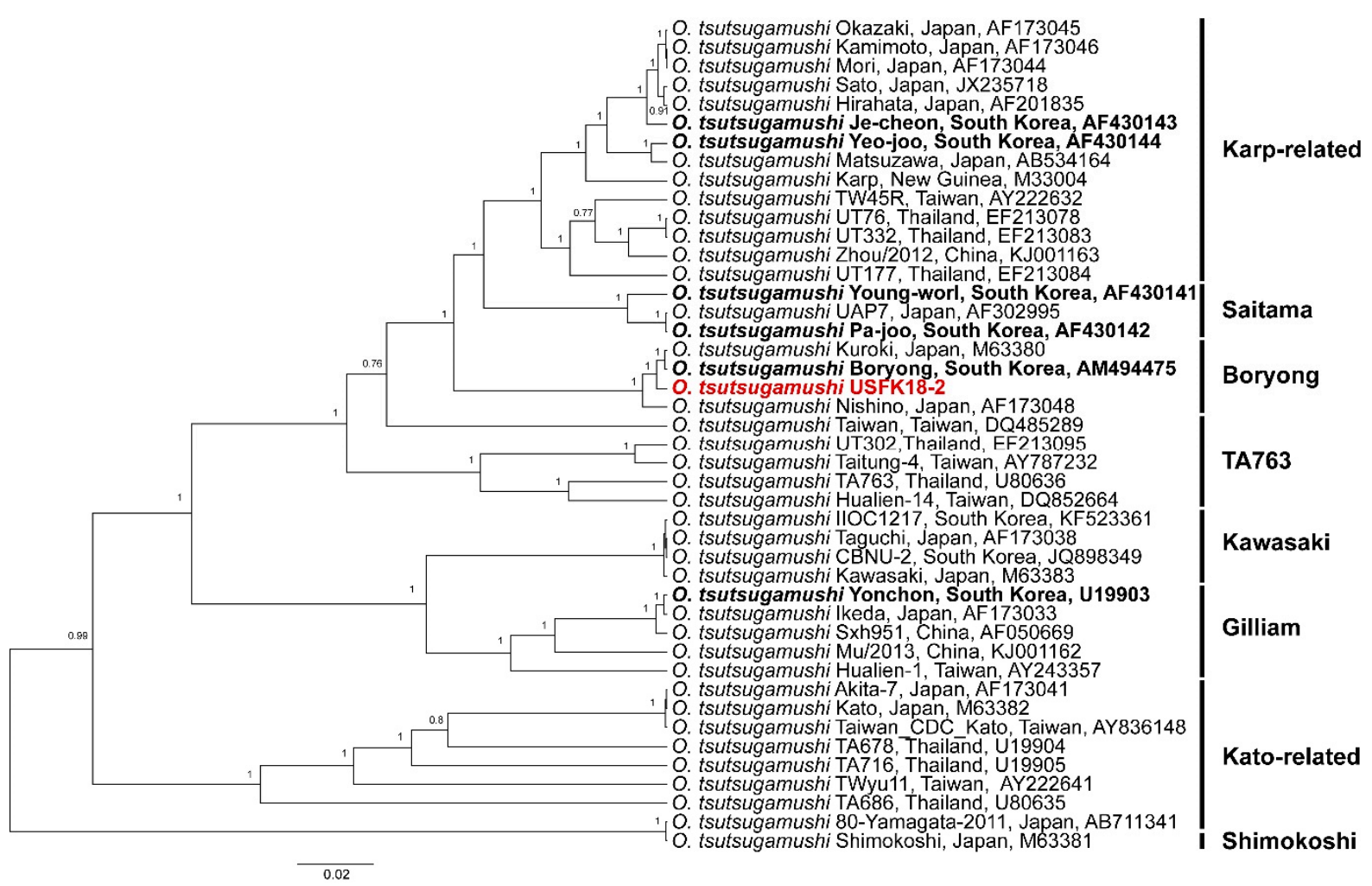

Figure 2. Phylogenetic analysis of the O. tsutsugamushi 56-kDa TSA gene identified from the USFK patient eschars. Phylogenetic relationships of the whole-genome sequences of O. tsutsugamushi 56-kDa TSA gene were inferred using Bayesian inference in BEAST (v1.10.4), using default priors and assuming homochromous tips. Upon running the Markov chain Monte Carlo analyses until adequate sample sizes (ESS > 200) were obtained, TreeAnnotator (v2.5.4) was used to summarize a maximum clade credibility tree from the posterior tree distribution, using a 10\% burn-in. The Korean serotypes, Je-cheon (AF430143), Yeo-joo (AF430144), Pa-joo (AF430142), Young-worl (AF430141), Boryong (NC009488), and Yonchon (U19903), are indicated in bold. Topologies were evaluated by bootstrap analysis of 1000 iterations. Numbers along branches are bootstrap values. The scale bar represents nucleotide substitutions per site. 
Table 2. Primer sequences used for multiplex PCR-based next-generation sequencing.

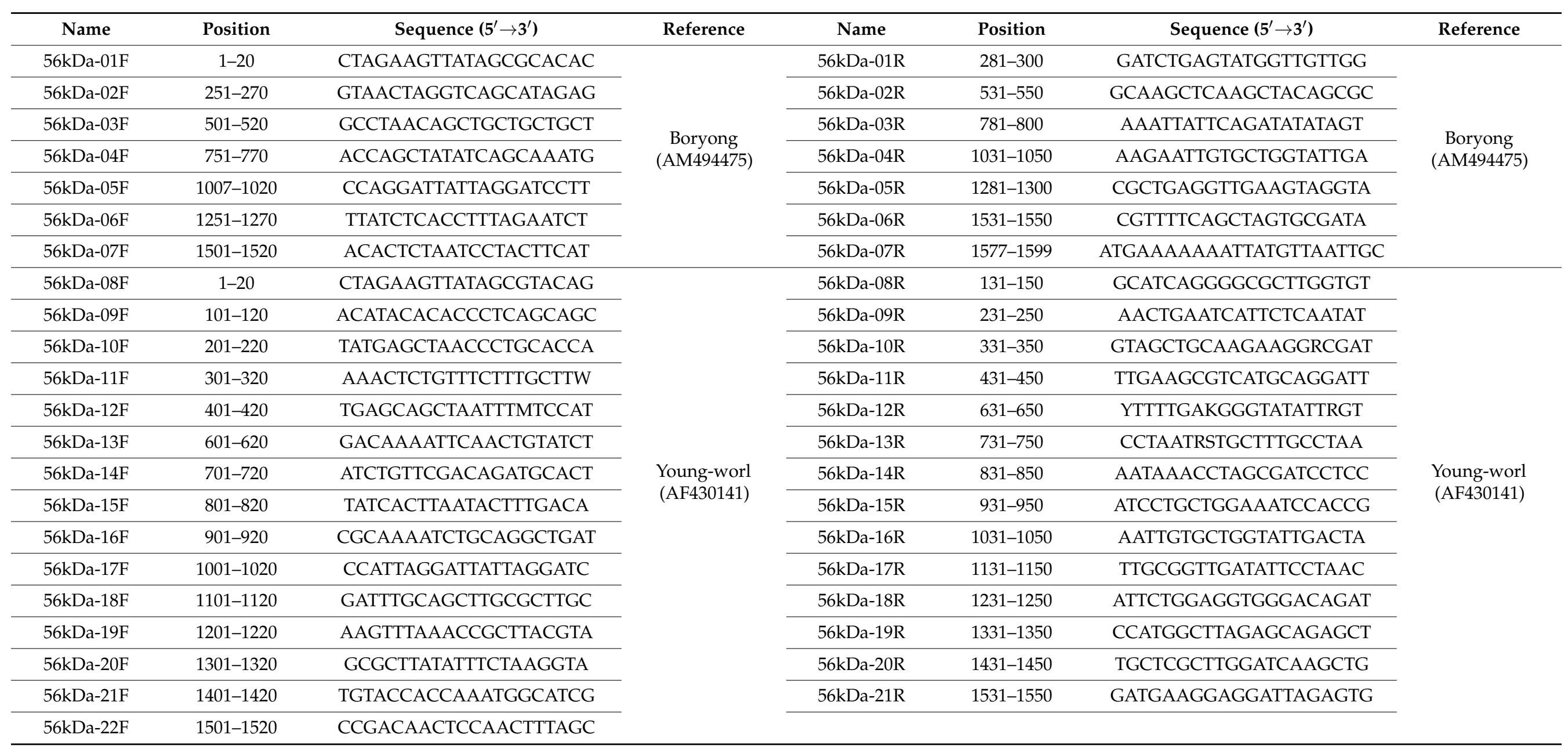




\section{Discussion}

Soldiers from ROK and the U.S. are exposed to rodents and their associated ectoparasites that may harbor zoonotic pathogens of military importance. Exposure to trombiculid mites that may be infected with $O$. tsutsugamushi increases potential disease risks during military operations at installations and training sites located throughout the Korean Peninsula. Investigations to identify infection rates of scrub typhus in USFK personnel have been carried out routinely through the comprehensive rodent field surveillance program [10]. While few scrub typhus cases (1 each year for 1995 and 2003, 2 for 2012, and 1 for 2013) were identified among US soldiers, it remains a serious health threat as it can quickly incapacitate large numbers of people and undermine military operations [11]. The serological survey of rodents, collected in the ROK, including the US operated military training sites located near the Demilitarized zone, demonstrated that the prevalence of $O$. tsutsugamushi showed a sero-positivity of $45.6 \%, 23.1 \%$, and $25.0 \%$ in Apodemus agrarius, Mus musculus, and Rattus norvegicus, respectively [12]. Therefore, continuous rodent trapping and surveillance are needed to identify disease risks in preventing the occurrence of patients with scrub typhus.

Using a multiplex PCR-based NGS strategy, we obtained the complete genomic sequence of 56-kDa TSA for O. tsutsugamushi for an eschar of the USFK patient. To investigate the emerging genotypes, a phylogenetic tree was constructed using the 56-kDa TSA full sequences. The result demonstrated that the USFK patient was infected with the Boryong genotype of $O$. tsutsugamushi, which is the most prevalent strain in the southwest part of ROK [6]. The clinical genotype of O. tsutsugamushi accounted for the Boryong strain of $79 \%$ and the Karp strain of $15 \%$, respectively, in ROK. Thus, we confirmed the USFK patient was most likely infected with the Boryong strain of O. tsutsugamushi.

In conclusion, the diagnosis and treatment of the USFK patient with scrub typhus were achieved by clarifying an eschar, clinical manifestations, and conducting an epidemiological survey. The multiplex-PCR-based NGS allowed for the delineation of the genotype of the clinical O. tsutsugamushi strain by acquiring the complete genomic sequence of 56-kDa TSA directly obtained from the clinical specimen. The clinical aspect of the USFK patient with scrub typhus and genetic identification of O. tsutsugamushi provides significant insights into clinical manifestations, epidemiological interviews, and high-throughput sequencing for the preparedness of the bacterial disease. This report raises awareness among physicians for the zoonotic disease, scrub typhus, especially in high endemic regions of the ROK.

\section{Materials and Methods}

\subsection{DNA Isolation from Eschar and Nested PCR}

Bacterial DNA was obtained from eschar by standard procedures using a High Pure PCR Template Preparation Kit (Roche, Basel, Switzerland). Nested PCR was performed in a $25 \mu \mathrm{L}$ reaction mixture containing $0.1 \mathrm{mM}$ dNTP Mix, 0.625 units TaKaRa Ex Taq polymerase (Takara, Shiga, Japan), $0.4 \mu \mathrm{M}$ of each primer, and $1.5 \mu \mathrm{L}$ template. Oligonucleotide primer sequences were Ri-A (outer): 5' -TTTCGAACGTGTCTTTAAGC-3', Ri-B (outer): 5'-ACAGATGCACTATTAGGCAA-3', Ri-E (inner): 5'-GTTGGAGGAATGATTACTGG-3', and Ri-F (inner): $5^{\prime}$-AGCGCTAGGTTTATTAGCAT-3' for the 56-kDa TSA gene [13]. The PCR conditions were: initial denaturation at $95^{\circ} \mathrm{C}$ for $5 \mathrm{~min}$, followed by 30 cycles of denaturation at $94{ }^{\circ} \mathrm{C}$ for $40 \mathrm{~s}$, annealing at $52{ }^{\circ} \mathrm{C}$ for $40 \mathrm{~s}$, elongation at $72{ }^{\circ} \mathrm{C}$ for $90 \mathrm{~s}$. Additionally, final elongation was done at $72{ }^{\circ} \mathrm{C}$ for $7 \mathrm{~min}$. PCR products were extracted using a PCR Purification Kit (Cosmo Genetech, Seoul, Korea), and DNA sequencing was performed in an Automatic Sequencer, ABI 3730XL DNA Analyzer (Applied Biosystems, Foster City, CA, USA).

\subsection{Multiplex PCR-Based Next-Generation Sequencing (NGS)}

Multiplex PCR primers were designed with 150 or 300 bps intervals using BioEdit Sequence Alignment Editor (Version 7.1.11). Each primer for the multiplex PCR of $O$. tsutsugamushi 56-kDa TSA gene was prepared and mixed (Table 2). The first PCR was performed in $25 \mu \mathrm{L}$ reaction mixture containing $12.5 \mu \mathrm{L} 2 \times$ Uh pre-mix, $1.0 \mu \mathrm{L}$ of genomic 
DNA template, $1.0 \mu \mathrm{L}$ of each primer mixture, and $10.5 \mu \mathrm{L}$ distilled water (DW). Initial denaturation was performed by a cycle of $95{ }^{\circ} \mathrm{C}$ for $15 \mathrm{~min}$, then 40 cycles of $95{ }^{\circ} \mathrm{C}$ for $20 \mathrm{~s}, 50{ }^{\circ} \mathrm{C}$ for $40 \mathrm{~s}, 72{ }^{\circ} \mathrm{C}$ for $1 \mathrm{~min}$, and a cycle of final elongation at $72{ }^{\circ} \mathrm{C}$ for $3 \mathrm{~min}$. The second PCR was performed in $25 \mu \mathrm{L}$ reaction mixture containing $12.5 \mu \mathrm{L} 2 \times$ Uh pre-mix, $1.0 \mu \mathrm{L}$ of the first PCR product, $1.0 \mu \mathrm{L}$ of each primer mixture, and $10.5 \mu \mathrm{L}$ DW. Initial denaturation was performed at $95^{\circ} \mathrm{C}$ for $15 \mathrm{~min}$, then 25 cycles of $95^{\circ} \mathrm{C}$ for $20 \mathrm{~s}, 50{ }^{\circ} \mathrm{C}$ for $40 \mathrm{~s}, 72{ }^{\circ} \mathrm{C}$ for $1 \mathrm{~min}$, and final elongation at $72{ }^{\circ} \mathrm{C}$ for $3 \mathrm{~min}$. DNA libraries were prepared using a TruSeq Nano DNA LT sample preparation kit (Illumina, San Diego, CA, USA) according to the manufacturer's instructions. The samples were mechanically sheared using an M220 focused ultrasonicator (Covaris, Woburn, MA, USA). The amplicon was size-selected, A-tailed, ligated with indexes and adaptors, and enriched. The libraries were sequenced using a MiSeq benchtop sequencer (Illumina) with $2 \times 150 \mathrm{bp}$ with a MiSeq reagent V2 (Illumina).

\subsection{Phylogenetic Analysis}

The genome sequence of the O. tsutsugamushi 56-kDa TSA gene was aligned using the Clustal W method with the Lasergene program, version 5 (DNASTAR). Phylogenetic analysis was conducted using the Bayesian inference method. Topologies were evaluated by bootstrap analysis of 1000 iterations.

Author Contributions: Conceptualization, S.C. and J.C.A.; investigation, S.C., J.C.A., W.-K.K.; clinical data curation, J.C.A., and T.A.K.; methodology, K.P. (Kkothanahreum Park), J.S.N., S.-H.L., K.P. (Kyungmin Park), and J.K.; validation, T.A.K., H.-C.K., and W.-K.K.; writing-original draft preparation, S.C. and J.C.A., and J.-W.S., supervision, J.-W.S.; funding acquisition, T.A.K., and J.-W.S. All authors have read and agreed to the published version of the manuscript.

Funding: This study was supported by the Agency for Defense Development (South Korea) (grant number: UE202026GD). Partial funding was provided by the Armed Forces Health Surveillance Branch, Global Emerging Infections Surveillance and Response System (AFHSB-GEIS), Silver Spring, MD (ProMIS ID \#P0025-2016-2018-ME) and the 65th Medical Brigade, Camp Humphreys, Republic of Korea. The opinions expressed herein are those of the authors and are not to be construed as official or reflecting the views of the U.S. Department of the Army or Defense. Authors, as employees of the U.S. Government (J.C.A., T.A.K., H.-C.K.), conducted the work as part of their official duties. Title 17 U.S.C. $\$ 105$ provides that 'Copyright protection under this title is not available for any work of the United States Government.' 'Title 17 U.S.C. §101 defines U.S. Government work as work prepared by an employee of the U.S. Government as part of the person's official duties.'

Institutional Review Board Statement: Not applicable.

Informed Consent Statement: Informed consent was obtained from all subjects involved in the study.

Data Availability Statement: All data are included in the manuscript. The sequence generated during the current study is available at GenBank (accession number: MT989475).

Conflicts of Interest: The authors declare no conflict of interest.

\section{References}

1. Ghorbani, R.P.; Ghorbani, A.J.; Jain, M.K.; Walker, D.H. A Case of scrub typhus probably acquired in Africa. Clin. Infect. Dis. 1997, 25, 1473-1474. [CrossRef] [PubMed]

2. Maina, A.N.; Farris, C.M.; Odhiambo, A.; Jiang, J.; Laktabai, J.; Armstrong, J.; Holland, T.; Richards, A.L.; O’Meara, W.P. Q fever, scrub typhus, and rickettsial diseases in children, Kenya, 2011-2012. Emerg. Infect. Dis. 2016, 22, 883-886. [CrossRef] [PubMed]

3. Weitzel, T.; Dittrich, S.; López, J.; Phuklia, W.; Martinez-Valdebenito, C.; Velásquez, K.; Blacksell, S.D.; Paris, D.H.; Abarca, K. Endemic scrub typhus in South America. N. Engl. J. Med. 2016, 375, 954-961. [CrossRef] [PubMed]

4. Lee, H.-W.; Cho, P.Y.; Moon, S.-U.; Na, B.-K.; Kang, Y.-J.; Sohn, Y.; Youn, S.-K.; Hong, Y.; Kim, T.-S. Current situation of scrub typhus in South Korea from 2001-2013. Parasites Vectors 2015, 8, 1-4. [CrossRef] [PubMed]

5. Kelly, D.J.; Fuerst, P.A.; Ching, W.; Richards, A.L. Scrub Typhus: The geographic distribution of phenotypic and genotypic variants of Orientia tsutsugamushi. Clin. Infect. Dis. 2009, 48, S203-S230. [CrossRef] [PubMed] 
6. Stover, C.K.; Marana, D.P.; Carter, J.M.; Roe, B.A.; Mardis, E.; Oaks, E.V. The 56-kilodalton major protein antigen of Rickettsia tsutsugamushi: Molecular cloning and sequence analysis of the sta56 gene and precise identification of a strain-specific epitope. Infect. Immun. 1990, 58, 2076-2084. [CrossRef] [PubMed]

7. Park, J.W.; Jung, S.H.; Ha, D.R.; Park, D.W.; Lee, J.M.; Kim, S.H.; Jeong, H.J.; Kim, N.M.; Gill, B.C.; Lee, J.Y.; et al. Molecular epidemiology of an Orientia tsutsugamushi gene encoding a 56-kDa type-specific antigen in chiggers, small mammals, and patients from the southwest region of Korea. Am. J. Trop. Med. Hyg. 2018, 98, 616-624. [CrossRef] [PubMed]

8. No, J.S.; Kim, W.-K.; Cho, S.; Lee, S.-H.; Kim, J.-A.; Lee, D.; Song, D.H.; Gu, S.H.; Jeong, S.T.; Wiley, M.R.; et al. Comparison of targeted next-generation sequencing for whole-genome sequencing of Hantaan orthohantavirus in Apodemus agrarius lung tissues. Sci. Rep. 2019, 9, 1-9. [CrossRef]

9. Kim, W.-K.; No, J.S.; Lee, D.; Jung, J.; Park, H.; Yi, Y.; Kim, J.-A.; Lee, S.-H.; Kim, Y.; Park, S.; et al. Active targeted surveillance to identify sites of emergence of hantavirus. Clin. Infect. Dis. 2019, 70, 464-473. [CrossRef] [PubMed]

10. Yi, K.S.; Chong, Y.; Covington, S.C.; Donahue, B.J.; Rothen, R.L.; Rodriguez, J.; Arthur, J.D. Scrub typhus in Korea: Importance of early clinical diagnosis in this newly recognized endemic area. Mil. Med. 1993, 158, 269-273. [CrossRef] [PubMed]

11. O'Guinn, M.L.; Klein, T.A.; Lee, J.S.; Richards, A.L.; Kim, H.-C.; Ha, S.J.; Shim, S.H.; Baek, L.J.; Song, K.-J.; Chong, S.-T.; et al. Serological surveillance of scrub typhus, murine typhus, and leptospirosis in small mammals captured at firing points 10 and 60 , Gyeonggi Province, Republic of Korea, 2001-2005. Vector-Borne Zoonotic Dis. 2010, 10, 125-133. [CrossRef] [PubMed]

12. Kim, H.C.; Lee, I.Y.; Chong, S.T.; Richards, A.L.; Gu, S.H.; Song, J.-W.; Lee, J.S.; Klein, T.A. Serosurveillance of scrub typhus in small mammals collected from military training sites near the DMZ, northern Gyeonggi-do, Korea, and analysis of the relative abundance of chiggers from mammals examined. Korean J. Parasitol. 2010, 48, 237-243. [CrossRef] [PubMed]

13. Enatsu, T.; Urakami, H.; Tamura, A. Phylogenetic analysis of Orientia tsutsugamushi strains based on the sequence homologies of 56-kDa type-specific antigen genes. FEMS Microbiol. Lett. 1999, 180, 163-169. [CrossRef] [PubMed] 